\section{Is it necessary to use three mandatory loading doses when commencing therapy for neovascular age-related macular degeneration using bevacizumab? (BeMOc Trial)}

G Menon', M Chandran', S Sivaprasad², R Chavan ${ }^{3}$, N Narendran ${ }^{3}$ and $Y$ Yang $^{3}$

\begin{abstract}
Purpose To determine whether a Pro Re Nata (PRN) regimen with three initial mandatory loading doses results in better functional and anatomical outcome compared with a PRN regimen without initial loading when using intravitreal bevacizumab in patients with minimal classic or occult choroidal neovascularisation secondary to age-related macular degeneration. Methods Patients were randomised (1:1) to Loading (LD group) or No Loading (NLD group) and treated with open label intravitreal bevacizumab. In the LD group, patients received two mandatory doses after the baseline dose before entering the PRN phase and in the NLD group, patients did not receive mandatory doses after the baseline dose. Six-weekly evaluations were performed up to week 54 and retreatment was done based on OCT criteria. Visual stability and reduction in central retinal thickness were compared between groups. Results 49 patients were in the NLD group and 50 patients were in the LD group. At the 12-month end point, $84 \%$ of the patients in the LD group achieved visual stability ( $<15$ letter loss) compared with $67 \%$ of the patients in the NLD group $(P<0.05)$. The mean reduction in central macular thickness was $105.35 \mu \mathrm{m}$ in the LD group and $81.45 \mu \mathrm{m}$ in the NLD group $(P>0.05)$. There was no significant difference in scores of VFQ-25
\end{abstract}

questionnaire testing between the two groups and no serious ocular or systemic side effects were observed.

Conclusion The results supported our hypothesis that a loading dose leads to slightly better visual stability in terms of proportions of patients experiencing moderate visual loss, but did not support the hypothesised difference in anatomical outcome.

Eye (2013) 27, 959-963; doi:10.1038/eye.2013.93; published online 7 June 2013

Keywords: bevacizumab; loading dose; age-related macular degeneration; choroidal neovascularisation

\section{Introduction}

The efficacy of intravitreal bevacizumab, used on an off-label basis, for the treatment of neovascular age-related macular degeneration (nAMD) has been extensively investigated in the last few years. ${ }^{1,2}$ In the IVAN study, patients in all treatment arms received three injections of either ranibizumab or bevacizumab from baseline, but in the CATT study protocol, patients in both Pro Re Nata (PRN) arms did not have any mandatory loading doses after the baseline dose of either ranibizumab or bevacizumab. In the CATT study, patients in the PRN arm of bevacizumab (no mandatory
${ }^{1}$ Frimley Park NHS Foundation Trust, Frimley, UK

${ }^{2}$ Kings College Hospital NHS Foundation Trust, London, UK

${ }^{3}$ Royal Wolverhampton Hospitals NHS Trust, Wolverhampton, UK

Correspondence: G Menon, Frimley Park NHS Foundation Trust, Portsmouth Road, Frimley, Surrey GU16 7UJ, UK Tel: +44 (0)1276604838; Fax: +44 (0)1276604149. E-mail: geeta.menon@ fph-tr.nhs.uk

Received: 15 February 2013 Accepted in revised form: 14 April 2013 Published online: 7 June 2013 
loading doses) had lower, albeit statistically non-inferior, mean visual acuity scores and less reduction in retinal thickness compared with patients in the continuous monthly fixed dosing bevacizumab arm at the 12-month primary end point analysis. ${ }^{1,2}$ These observations have led to the widely held view that it is necessary to administer a continuous regimen especially in the first few months of therapy when treating

nAMD with bevacizumab. 1,3,4

In this study, we tested the hypothesis that a PRN regimen with three initial mandatory doses should result in better functional and anatomical outcome compared with a PRN regimen without any mandatory loading when using intravitreal bevacizumab to treat patients with minimal classic or occult choroidal neovascularisation secondary to age-related macular degeneration.

\section{Materials and methods}

The study protocol (EUDRACT No: 2006-003033-33, ISRCTN number: 12980412) was approved by the Institutional Local Ethics Committee and was carried out in accordance with the Declaration of Helsinki. Owing to the off-label use of bevacizumab being approved within one hospital trust, a reasonable and pragmatic sample size of 100 patients was selected to enable the study to be carried out as a monocentric study. Eligible criteria included treatment-naive patients with active subfoveal choroidal neovascularisation of minimally classic or occult type, secondary to age-related macular degeneration, confirmed on fluorescein angiography, and no other visually significant ocular pathology. All patients at baseline were subjected to visual acuity measurement, slit lamp examination, dilated biomicroscopy, Goldmann tonometry and OCT examination. Patients were randomised $(1: 1)$ to receive either one injection of bevacizumab at baseline followed by a PRN regimen (No Loading Dose-NLD group) or three 6-weekly injections of bevacizumab followed by PRN (Loading Dose-LD group). Bevacizumab aliquots were procured from the compounding pharmacy at Moorfields Eye Hospital, London, UK, and administered without masking by intravitreal injections $(1.25 \mathrm{mg} /$ $0.05 \mathrm{ml}$ ) with a 30-gauge needle and a $1-\mathrm{ml}$ tuberculin syringe under aseptic conditions. Patients were followed up every 6 weeks up to week 54 . At each visit, the patients were assessed for best corrected visual acuity, central macular thickness (CMT) on OCT and also adverse events. Best corrected visual acuity scores were measured following full subjective refraction using modified ETDRS (logMAR) charts. Time domain OCT with Stratus OCT (Version 6, Carl Zeiss Meditec International, Welwyn Garden City, UK) was used throughout the study. Patient-reported outcome measures were determined using the VFQ-25 questionnaire tool at baseline and week 54. In the PRN phase, retreatment was administered if CMT exceeded $250 \mu \mathrm{m}$. Comparison between groups for statistically significant differences in visual acuity and CMT was carried out at study end point of week 54 (unpaired $t$-test and $\chi^{2}$ test). Descriptive statistics were used for injection numbers and adverse events.

\section{Results}

Out of 100 enrolled patients, 99 completed 1-year followup. Data analysis was based on the per protocol set of 99 study eyes of 99 patients. Forty-nine patients (73.5\% क ) received one initial injection followed by PRN (NLD group) and 50 patients $(72 \%$ ㅇ) received three loading injections followed by a PRN regimen (LD group). The baseline parameters of patients in both groups are shown in Table 1. The two groups were balanced at baseline in terms of mean visual acuities and mean CMT. Patients in the LD group received a mean of 5.8 injections (3-9) and patients in the NLD group received a mean of 4.7 injections (1-9). Out of the 49 patients in the NLD group, 18 patients only required two injections in the first three visits and 4 patients required only one injection in the first three visits.

At week 54 primary end point analysis, the proportions of patients with visual stability, defined as $\leq 15$ letter loss from baseline, were $84 \%$ in the LD group compared with only $67 \%$ in the NLD group (Figure 1 ). This difference was statistically significant (one-sided $\chi^{2}$ test, $\left.\chi^{2}=3.51, P<0.05\right)$. Patients in the NLD group had mean visual acuity gain of 0.86 letters and patients in the LD group had a mean visual acuity gain of 2.08 letters $(P>0.05)$. The percentage of patients who gained 10 or more letters was higher in the LD group (28\%) compared with the NLD group (26.3\%), but this difference was not statistically significant.

Table 1 Distribution of gender, age and mean values of visual acuity and CMT at baseline

\begin{tabular}{lcc}
\hline & $\begin{array}{c}\text { Loading group } \\
(\mathrm{n}=50), n(\%)\end{array}$ & $\begin{array}{c}\text { No Loading group } \\
(\mathrm{n}=49), n(\%)\end{array}$ \\
\hline Men & $14(28)$ & $13(26.5)$ \\
Women & $36(72)$ & $36(73.5)$ \\
Age 61-70 & $5(10)$ & $8(16.3)$ \\
Age 71-80 & $22(44)$ & $13(26.5)$ \\
Age 81+ & $23(46)$ & $28(57)$ \\
Visual acuity (letters) & $45.52(25-75)$ & $48.57(23-77)$ \\
mean (range) & & \\
20-40 letters & 22 & 16 \\
41-60 letters & 20 & 24 \\
61-80 letters & 8 & 9 \\
Mean CMT $(\mu \mathrm{m})$ & 311.18 & 355.89 \\
\hline
\end{tabular}




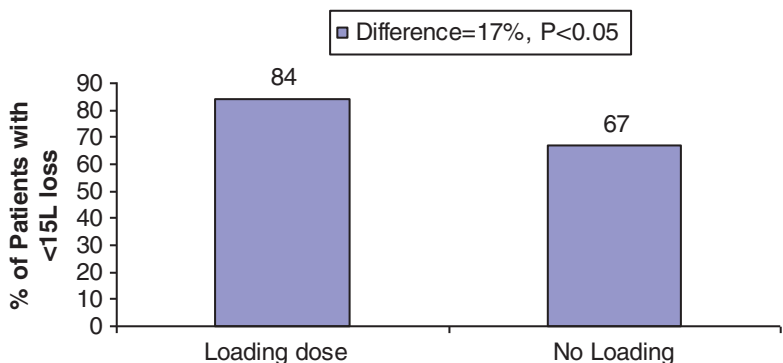

Figure 1 Histogram plot of percentages of patients with $<15$ letter loss in each group.

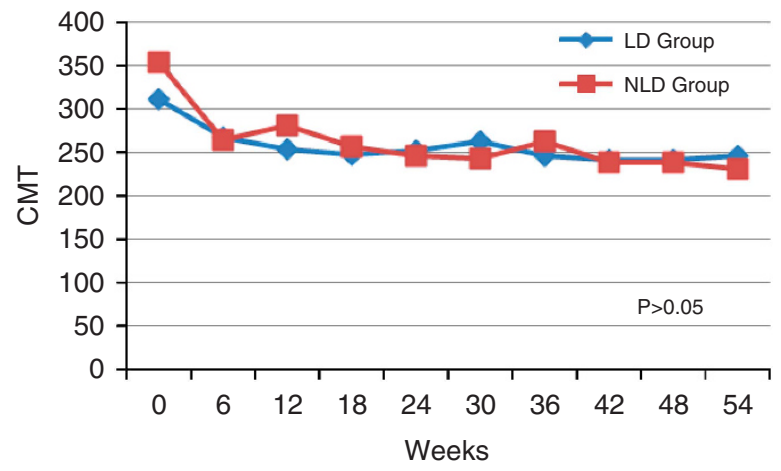

Figure 2 Reduction in CMT $(\mu \mathrm{m})$ over 54 weeks.

There was a reduction in CMT from baseline to week 54 in both groups (Figure 2). Last observation carried forward was used for any missing data. The mean reduction was $81.45 \mu \mathrm{m}$ in the NLD group and $105.35 \mu \mathrm{m}$ in the LD group. When controlling for the baseline thickness (one-way analysis of covariance), the reductions were highly significant within each group $(P<0.0001)$, but there was no significant difference between groups in terms of mean change in CMT from baseline ( $P>0.05$, unpaired $t$-test). The sharpest reduction in CMT occurred after the first injection, between baseline and week 6 (groups were combined for this analysis), with a mean reduction of $67.68 \mu \mathrm{m}$ before reaching asymptote. There was no significant difference between the two groups in the change in scores from baseline to week 54 in all of the 12 domains of VFQ-25 questionnaire testing. The overall mean scores for NLD were 3.05 and 3.01 at baseline and week 54, respectively. The overall mean scores for LD were 3.02 and 3.08 at baseline and week 54, respectively.

There were no serious ocular adverse events in either group. In total, there were six treatment-emergent adverse events in the LD group and five in the NLD group. This difference was judged to be not significant and not tested statistically due to the small numbers. One patient in the LD group developed myocardial ischaemia during the study and was withdrawn from the study.
Table 2 Treatment-emergent adverse events

\begin{tabular}{lcc}
\hline Event & $\begin{array}{c}\text { Loading } \\
\text { group, } n(\%)\end{array}$ & $\begin{array}{c}\text { No Loading } \\
\text { group, } n(\%)\end{array}$ \\
\hline Conjunctivitis & $2(4)$ & $1(2)$ \\
Glaucoma & $1(2)$ & 0 \\
Hordeolum internum & 0 & $1(2)$ \\
Subconjunctival haemorrhage & $1(2)$ & 0 \\
Corneal abrasion & 0 & $1(2)$ \\
Respiratory tract infection & 0 & $1(2)$ \\
Fall and radius fracture & 0 & $1(2)$ \\
Dizziness & $1(2)$ & 0 \\
Trabeculectomy & $1(2)$ & 0 \\
Myocardial ischaemia & $1(2)$ & 0 \\
\hline
\end{tabular}

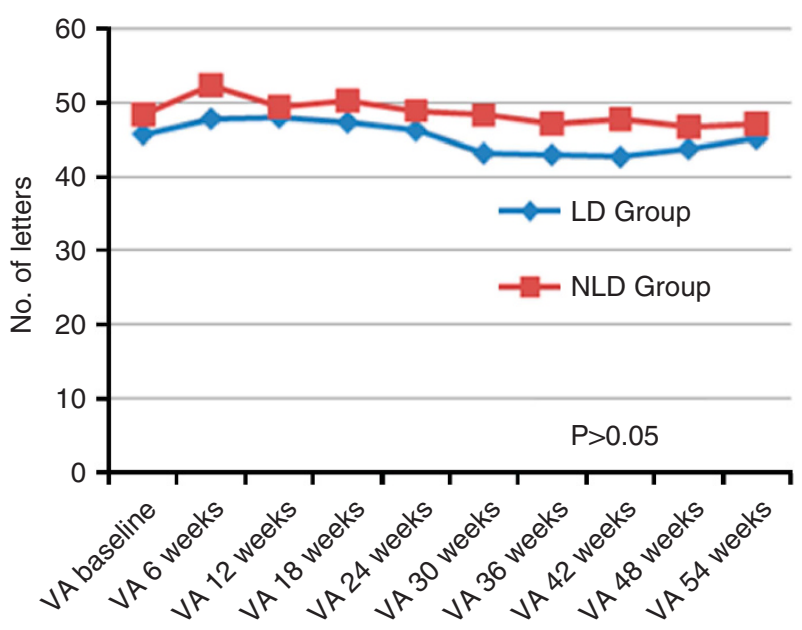

Figure 3 Mean visual acuity (no of letters) during study period.

This particular event was felt to be treatment-related. Table 2 shows the adverse events in both groups.

\section{Discussion}

Overall, patients who received three loading doses had better outcomes in terms of mean visual acuity, proportion with visual stability and reduction in macular thickness. However, when comparing the two groups statistically, only the comparison of the difference in proportions of patients with visual stability was found to be just significant at the 0.05 level with a one-sided $\chi^{2}$ test. A post hoc analysis comparing the two groups in terms of the mean visual scores turned out to be not statistically significant (Figure 3). This is most likely to be due to the inevitably small expected difference between the two regimens, but also partly due to the small sample size. It is possible that fewer injections in the NLD group during the early stages made a slight difference in visual outcome in a proportion of patients and this was reflected in the analysis of stability, but not in terms of mean scores in BCVA. Also, the Loading group had more 
patients in the lowest acuity stratum and a floor effect may have contributed to the better stability outcome in this group.

Our results are comparable to other studies that have compared treatment regimens with and without loading doses. Arias et $a l^{3}$ reported better visual outcome in their loading group compared with no loading at 6 months in a non-randomised study using bevacizumab in AMD, but the sample size was smaller $(n=50)$ and consisted of a high proportion of eyes with prior PDT. Gupta et $a l^{5}$ in a retrospective, non-randomised study in 2010 comparing two regimens of ranibizumab in a similar way also found better visual outcome in their loading group. It does appear therefore that a PRN regimen of bevacizumab or ranibizumab tends to give a better visual outcome in the short term at least when there are fixed loading doses initially. However, two studies have so far shown that a loading dose may not be necessary. In the CATT study, the PRN arms had no loading dose and still produced non-inferior visual outcomes to the monthly fixed dosing arms. This could be due to the low threshold for retreatment when any fluid or RPE detachment on OCT were criteria for retreatment. ${ }^{2}$ More recently, El-Mollayess et $a l^{6}$ showed that there was no difference in visual outcome between a fixed dosing regimen of bevacizumab compared with a PRN regiment without any loading doses. Again there was a revised retreatment protocol with a lower threshold for retreatment in their study that excluded patients with RAP lesions, which tend to require more frequent retreatment.

Another observation from our study was that despite the similarly significant reductions in macular thicknesses, and the similar overall numbers of injections by week 54 in both groups, there was still a detectable difference in visual outcome between the groups, with the LD group performing better. This supports the view that prompt control of neovascular activity and leakage in the early phases of treatment leads to better visual outcome. ${ }^{7-10}$ Although the amount of difference in visual outcomes between the two groups were small, this may still be clinically relevant, as small differences can be important especially when acuities are moderately good. ${ }^{10,11}$

At the week 54 end point visit, there was only a modest amount of improvement in acuity compared with baseline in both groups. This could be due to a bias in the case mix of quite advanced lesions, which were minimal classic and occult-only lesions and with mean baseline acuity level of around 20/160. An additional factor could be the 6-weekly injection interval giving a slightly less sharp rise in visual recovery in the initial months of commencing treatment, as seen in other studies with less frequent injections. ${ }^{12,13}$ The OCT retreatment criteria of $250 \mu \mathrm{m}$ used in this study was probably not aggressive enough. The reasons we used a 6-weekly interval and a retreatment threshold of $250 \mu \mathrm{m}$ was related to the lack of experience of using avastin at the time of the study was designed especially for occult and minimally classic lesions and the prevailing tendency to follow the retreatment criteria from the PrONTO study. ${ }^{14}$ The study design preceded the CATT study that eventually confirmed the safety of a 4-weekly protocol and a more aggressive retreatment policy. It is now more widely accepted that retreatment should be administered more frequently at 4-weekly intervals for intraretinal and subretinal signs of fluid leakage on OCT, even if the macular thickness is not $>250 \mu \mathrm{m} .4,15,16$

To our knowledge, this is the first prospective, randomised controlled study to compare multiple outcome measures of a loading regimen with a nonloading regimen when commencing intravitreal bevacizumab therapy for neovascular macular degeneration. The results supported our hypothesis that a loading dose leads to slightly better visual outcome, but did not support the hypothesised difference in anatomical outcome nor an outcome of quality of life scores. The gain in visual outcome by administering a loading regimen was small but probably clinically relevant and justified, as the use of a loading regimen does not increase the overall number of injections in the first year.

\section{Summary}

What was known before

- Intravitreal bevacizumab is used to treat choroidal neovascularisation secondary to AMD.

\section{What this study adds}

- The results supported our hypothesis that a loading dose leads to slightly better visual outcome. It did not support the hypothesised difference in anatomical outcome.

\section{Conflict of interest}

The authors declare no conflict of interest.

\section{References}

1 Chakravarthy U, Harding SP, Rogers CA, Downes SM, Lotery AJ, Wordsworth $\mathrm{S}$ et al. Ranibizumab versus bevacizumab to treat neovascular age-related macular degeneration: one-year findings from the IVAN randomized trial. Ophthalmology 2012; 119: 1399-1411.

2 CATT Research Group. Ranibizumab and bevacizumab for neovascular age-related macular degeneration. $\mathrm{N}$ Engl J Med 2011; 364: 1897-1908.

3 Arias L, Caminal JM, Casas L, Masuet C, Badia MB, Rubio $\mathrm{M}$ et al. A study comparing two protocols of 
treatment with intravitreal bevacizumab (Avastin) for neovascular age-related macular degeneration. Br J Ophthalmol 2008; 92: 1636-1641.

4 Martin DF, Maguire MG, Fine SL, Ying GS, Jaffe GJ, Grunwald JE et al. Comparison of age-related macular degeneration treatments trials (catt) research group, ranibizumab and bevacizumab for treatment of neovascular age-related macular degeneration: two year results. Ophthalmology 2012; 119: 1388-1398.

5 Gupta B, Adewoyin T, Patel SK, Sivaprasad S. Comparison of two intravitreal ranibizumab treatment schedules for neovascular age-related macular degeneration. Br J Ophthalmol 2011; 95(3): 386-390.

6 El-Mollayess G, Mahfoud Z, Schakal A, Salti H, Jaafar D, Bashshur ZF. Fixed-interval versus OCT-guided variable dosing of intravitreal bevacizumab in the management of neovascular age-related macular degeneration: A 12-month randomized prospective study. Am J Ophthalmol 2012; 153: 481-489.

7 Brown DM, Kaiser PK, Michels M, Soubrane G, Heier JS, Kim RY et al. ANCHOR Study Group. Ranibizumab versus verteporfin for neovascular age-related macular degeneration. N Engl J Med 2006; 355(14): 1432-1444.

8 Singer MA, Awh CC, Sadda S, Freeman WRAntoszyk AN, Wong P, Tuomi L. HORIZON: an open-label extension trial of ranibizumab for choroidal neovascularization secondary to age-related macular degeneration. Ophthalmology 2012; 119: $1175-1183$.

9 Olajumoke S, Gupta B, Vemala R, Sivaprasad S. Visual acuity outcomes in ranibizumab-treated neovascular age-related macular degeneration; stratified by baseline vision. Clin Exp Ophthalmol 2011; 39: 3-5.
10 Williams TA, Blyth CP. Outcome of ranibizumab treatment in neovascular age related macular degeneration. Eye 2011; 25: 1617-1621.

11 Rathore D, Oyede T, Narendran N, Yang YC. Snellen versus $\log \mathrm{MAR}$ visual acuity charts for evaluating driving standards in patients with neovascular macular degeneration. Br J Vis Impair 2012; 30: 160-167.

12 Schmidt-Erfurth U, Eldem B, Guymer R, Korobelnik JF, Schlingemann RO, Axer-Siegel $\mathrm{R}$ et al. EXCITE Study Group. Efficacy and safety of monthly versus quarterly ranibizumab treatment in neovascular age-related macular degeneration: the EXCITE study. Ophthalmology 2011; 118(5): 831-839.

13 Tufail A, Patel PJ, Egan C, Hykin P, da Cruz L, Gregor $\mathrm{Z}$ et al. ABC Trial Investigators. Bevacizumab for neovascular age related macular degeneration (ABC Trial): multicentre randomised double masked study. BMJ 2010; 340: c2459.

14 Fung AE, Lalwani GA, Rosenfeld PJ, Dubovy SR, Michels S, Feuer WJ et al. An optical coherence tomography-guided, variable dosing regimen with intravitreal ranibizumab (Lucentis) for neovascular age-related macular degeneration. Am J Ophthalmol 2007; 143(4): 566-583.

15 Heier JS, Brown DM, Chong V, Korobelnik JF, Kaiser PK, Nguyen QD et al. VIEW 1 and VIEW 2 study groups. Intravitreal aflibercept (VEGF trap-eye) in wet age-related macular degeneration. Ophthalmology 2012; 119: 2537-2548.

16 Suner IJ, Yau L, Lai P. HARBOR study: one-year results of efficacy and safety of $2.0 \mathrm{mg}$ versus $0.5 \mathrm{mg}$ ranibizumab in patients with subfoveal choroidal neovascularization secondary to age-related macular degeneration. Paper presented at The Association for Research in Vision and Ophthalmology Annual Meeting, 1 May 2011, Fort Lauderdale, FL (abstract no. 3677) 